\title{
Time-Resolved Force Measurements to Determine Positioning Tolerances for Impulse-Based Indentations
}

\author{
Tobias Valentino $^{1}$ D Marian Höhmann ${ }^{1} \cdot$ Kevin Schünemann $^{1}$. \\ Jonas Wentzler ${ }^{1} \cdot$ Tim Wünderlich $^{1} \cdot$ Tim Radel $^{1}$ · Frank Vollertsen ${ }^{1,2}$
}

Accepted: 2 March 2021 / Published online: 31 May 2021

(c) The Author(s) 2021

\begin{abstract}
High-throughput experimentation methods determine characteristic values, which are correlated with material properties by means of mathematical models. Here, an indentation method based on laser-induced shock waves is presented, which predicts the material properties, such as hardness and tensile strength, by the induced plastic deformation in the substrate material. The shock wave pushes a spherical indenter inside a substrate material. For reproducible indentations, the applied load is of importance. To compare different processes and process parameters, the measured plastic deformation is normalized by the applied load. However, eccentric irradiation leads to altered beam profiles on the surface of spherical indenters and the angle of incidence is changed. Thus, the influence of eccentric irradiation is studied with an adapted time-resolved force measurement setup to determine the required positioning tolerances. The spherical indenter is placed inside a cylindrical pressure cell to increase the laser-induced shock pressure. From the validated time-resolved force measurement method we derive that deviations from the indentation forces are acceptable, when the lateral deviation of the beam center, which depends only on the alignment of the setup, does not exceed $\pm 0.4 \mathrm{~mm}$. A vertical displacement from the focus position between $-3.0 \mathrm{~mm}$ and $+2.0 \mathrm{~mm}$ still leads to acceptable deviations from the indentation force.
\end{abstract}

Keywords Measuring instrument · Position accuracy $\cdot$ Positioning tolerances · Maximum indentation force $\cdot$ High-throughput indentation

Tobias Valentino

valentino@bias.de

1 BIAS - Bremer Institut Für Angewandte Strahltechnik GmbH, Klagenfurter Straße 5, 28359 Bremen, Germany

2 Universität Bremen, Bibliothekstraße 1, 28359 Bremen, Germany 


\section{Introduction}

The exact determination of the material properties of new alloys still requires numerous testing methods although computer aided predictions of the material properties exist since the 1970s [1]. Hence, cost-efficient, and high-throughput material development processes are needed to meet the demands to efficiently identify novel structural materials [2]. A possibility to decrease the processing time is to produce smaller samples instead of large casts [3]. However, high throughput material development does not only imply the fast production of new alloys. It also requires rapid material experimentation and characterization. Moreover, the constant urge for better and well-adapted material properties increases the complexity of materials and the scientific ability for the rational materials design. As a result of this complexity, high-throughput material development has been recognized as a new scientific approach to generate knowledge [4]. Instead of applying time-consuming conventional measurement methods, such as hardness or tensile tests, on macroscopic samples, the characterization is performed on small samples with suitable testing methods [5]. These testing methods determine characteristic values, which can be rapidly identified and correlated with material properties [6]. Nevertheless, the characteristics of these novel methods must be understood to be able to operate at the same accuracy level as existing methods.

Here, an indentation method is investigated, which is based on laser-induced shock waves. The novel laser-induced shock wave indentation method is further referred to as LiSE. Instead of using conventional indentation testing machines, a shock wave is induced on top of a spherical indenter with a high intensity pulsed transversely excited atmospheric-pressure (TEA)- $\mathrm{CO}_{2}$ laser. The pressure of the shock wave pushes the indenter within $20 \mu$ s inside the test sample and creates an indentation [7]. From the indentation profile, different characteristic values are determined. These values are correlated with mechanical material properties. So far, it has been shown that the measured characteristic values (indentation depth, indentation diameter and pile-up as well as sink-in behavior) with LiSE strongly correlate with the material hardness [8] and tensile strength [9]. Pre-treatment and ablation layers of the indenter are not necessary with the wavelength of the laser beam $(\lambda=10.6 \mu \mathrm{m})$ because a plasma is quasi-instantly formed on top of the irradiated surface, which absorbs nearly all the irradiation of the laser beam [10]. Although the plasma temperature increases up to 19,000 K [11] above the indenter during the indentation process, no significant heating of the tested substrate material is observed underneath the indenter [12]. When the intensity of the $\mathrm{CO}_{2}$ laser pulse exceeds a critical threshold $\left(>10^{7} \mathrm{~W} / \mathrm{cm}^{2}\right)$, the plasma results in a shock wave [13]. Barchukov et al. observed that the shock wave initiates approximately $5 \mathrm{~mm}$ above the metallic surfaces for pulsed $\mathrm{CO}_{2}$ laser [14]. Studies from Sai Shiva et al. showed that the shock wave transforms during propagation from planar, to cylindrical and finally to spherical [15].

To achieve high-throughput experimentation, several indentations must be induced into the substrate material in the shortest possible time. The shock waves 
can be generated by a scanner system above several indenters, which are located on one large sample or on many micro samples. The investigated TEA- $\mathrm{CO}_{2}$ laser has a repetition rate of $50 \mathrm{~Hz}$. When dealing with high-throughput processes, one challenge is the accurate positioning in the given time range. For the implementation of a high-throughput method, it is therefore necessary to identify the required positioning tolerances between the laser beam and the indenter. Eccentric irradiation leads to altered beam profiles on the surface of spherical indenters and the angle of the resulting force is changed. Thus, the reproducible application of a defined load is of great importance and the influence of eccentric irradiation should be investigated. We present and investigate a time-resolved measurement method for short-time indentations to determine the maximum indentation force and transferred momentum of the indenter and to derive acceptable positioning tolerances. We imply that a shift of focal position or shift in $\mathrm{x}$ - and $\mathrm{y}$-direction (lateral shift) is still acceptable, if the maximum force at the shifted position is still within the range of standard deviation of the maximum force at the reference position. This and the time-resolved measurement method are validated by conventional indentation tests, pendulum experiments, drop tests and LISE experiments.

\section{Methods}

\section{Indentation Experiments}

\section{Laser-Induced Shock Wave Indentation Method}

The experiments are conducted with a pulsed TEA-CO $\mathrm{CO}_{2}$ laser from SLCR. A maximum pulse energy of $6 \mathrm{~J}$ was used for the experiments. The temporal laser energy distribution measurements reveal a pulse duration of $100 \mathrm{~ns}$. A focusing mirror with a focal length of $200 \mathrm{~mm}$ is used, resulting in a minimal quadratic beam spot of $4 \mathrm{~mm}^{2}$ and in a laser intensity of $1.5 \mathrm{GW} / \mathrm{cm}^{2}$ at maximum pulse energy. The Rayleigh length of the laser beam is $z_{R}=10.8 \mathrm{~mm}$. The spatial energy distribution of the laser beam is a top-hat with a near-uniform fluence. The indentations were created with spherical $\mathrm{Al}_{2} \mathrm{O}_{3}$ indenter with an indenter radius of $R_{I}=1.5 \mathrm{~mm}$. A cylindrical pressure cell made of brass was used to increase the acting shock wave pressure on the indenter (see Fig. 1). Findings by Corsi et al. suggest that the reflected shock wave front can move faster in cylindrical cavities through the heated and accelerated air mass of the preceding direct shock wave [16]. Finally, this leads to a superposition of the preceding and the reflecting shock wave, which increases the acting shock wave pressure. Moreover, the pressure cell was designed according to the findings from [17] identifying the center of the shock wave above the surface. Thus, the cylindric pressure cell has a height of $10 \mathrm{~mm}$ and an inner diameter of $3.02 \mathrm{~mm}$. The indenter is placed inside the pressure cell.

Additionally, a reference setup without a pressure cell was investigated to compared time-resolved force measurements (see Fig. 2). The position unit was used for the reference setup without pressure cell. The mounting of the test samples is 


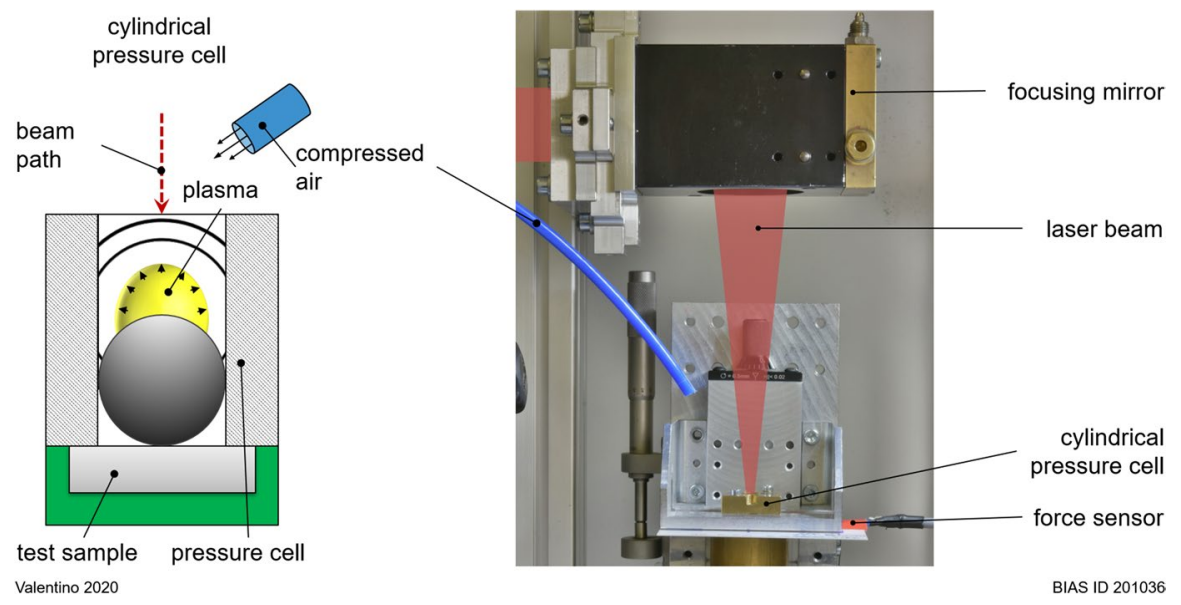

Fig. 1 Experimental setup with cylindrical pressure cell

based on the design guidelines for hardness testing according to [18]. The setups were flushed with compressed air to remove particles above the irradiated surface. Without a gas flushing of the pressure cell, the laser-induced plasma tends to be ignited randomly in mid-air, which lowers the pressure at the irradiated surface. The indentation experiments were conducted on Aluminum EN AW-1050 samples (further referred to as A199.5) with a $5 \mathrm{~mm}$ thickness and on steel S235 samples with a thickness of $3 \mathrm{~mm}$. The samples were embedded and prepared by grinding and polishing for the LiSE experiments and the Brinell hardness testing. For the LiSE experiments, the pulsed laser beam was irradiated on the indenters placed coaxially in the pressure cell and on top of the sample's surface. The position in focus
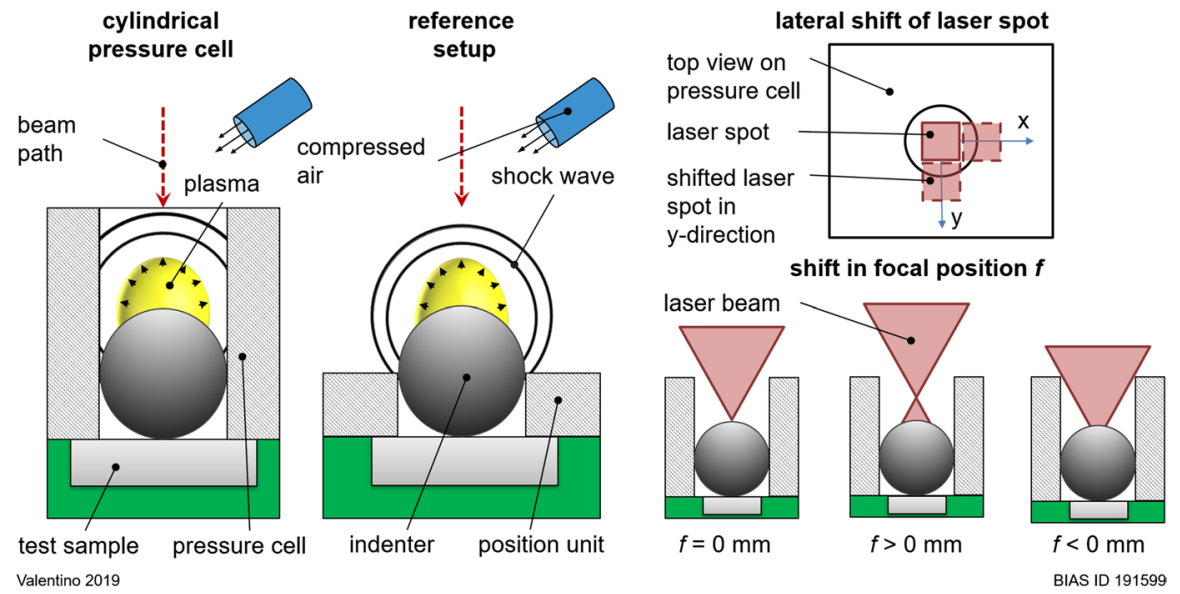

Fig. 2 Investigated pressure cell and conducted experiments on positioning tolerance 
is considered as the reference position. The conducted experiments on positioning tolerances are illustrated schematically in Fig. 2. The evaluation of the indentation diameter and indentation depth was carried out at $20 \times$ magnification with the microscope VK9710 from Keyence.

\section{Brinell Hardness}

The Brinell hardness of the samples was determined with a hardness testing machine from Struers. According to [19], an indentation load of $25 \mathrm{~N}$ was applied on a $1 \mathrm{~mm}$ indenter at a dwelling time of $15 \mathrm{~s}$. The A199.5 samples have a Brinell hardness of 35.5 HBW $1 / 2.5 \pm 0.2$ HBW $1 / 2.5$ and the steel S235 samples have a Brinell hardness of $105 \mathrm{HBW} 1 / 2.5 \pm 2 \mathrm{HBW} 1 / 2.5$. To compare the Brinell test results with the LiSE experiments, the measured indentation diameter $d_{i}$, the indenter diameter $\left(D_{I}=3 \mathrm{~mm}\right)$ and the maximum indentation force $F_{\max }$ are used to estimate the Brinell hardness. For spherical indenter, the correlation between the indentation diameter $d_{i}$ and the maximum indentation force $F_{\max }$ is given by the following equation:

$$
H B=\frac{0.102 \cdot 2 \cdot F_{\max }}{D_{I} \cdot \pi \cdot\left(D_{I}-\sqrt{{D_{I}^{2}}^{2}-d_{I}^{2}}\right)}
$$

\section{Conventional Compression Tests}

Conventional compression tests are performed on the Zwick/Roell Z250 stationary testing machine (see Fig. 3 left). This machine can apply a maximum load of $250 \mathrm{kN}$. The crosshead speed can be variably adjusted between $5 \cdot 10^{-5} \mathrm{~mm} / \mathrm{min}$ and $600 \mathrm{~mm} / \mathrm{min}$. For the indentation experiments the crosshead speed is set to $0.1 \mathrm{~mm} /$ min to allow a quasistatic indentation. The quasistatic indentation test is based on the Brinell hardness testing method. For the conventional indentation tests a spherical indenter is attached to a die (see Fig. 3 right). The positioning accuracy of the crosshead is $\pm 2 \mu \mathrm{m}$. For the compression tests a load cell from Zwick/Roell with a maximum test load of $200 \mathrm{~N}$ is used. The maximum deviation of the measured value is $0.25 \%$ above an applied load of $0.8 \mathrm{~N}$.

\section{Drop Tests}

Drop tests are performed to determine whether the necessary forming energy to create indentations is comparable to the LiSE experiments. The drop height $h$ is changed to influence the potential energy $E_{P o t}$ and accordingly, the forming energy. The drop tests setup is described in more detail in [20]. An increasing drop height may cause spinning of the indenter. Thus, the impact velocity in dependence of the drop height is verified with the high-speed camera Phantom V5.1 from Vision 


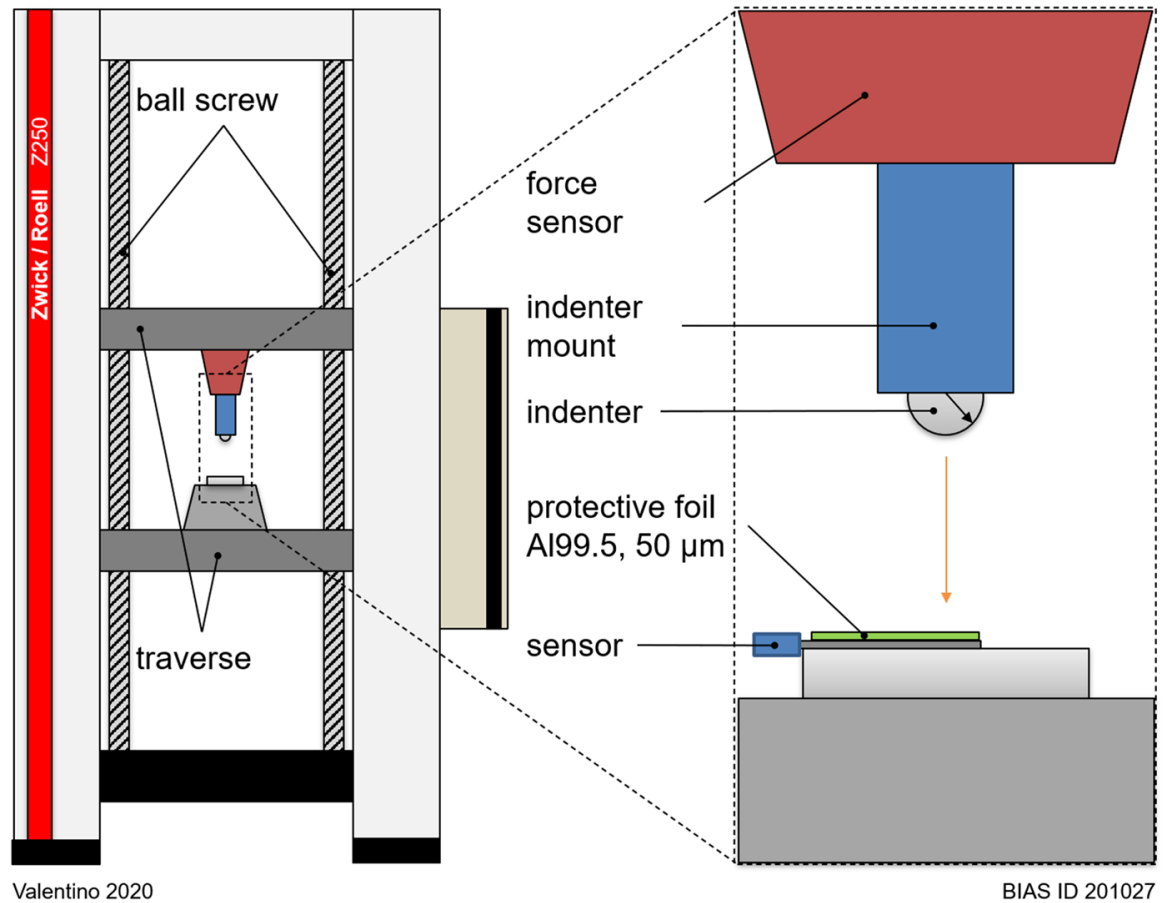

Fig. 3 Design of the tension-compression testing machine Z250 for validation of the measured forces with piezo sensor

Research. A frame rate of $1000 \mathrm{fps}$ with an exposure time of $990 \mu \mathrm{s}$ is used. The indentation results are compared with LiSE and the derived forming energy.

$$
E_{P o t}=m_{G} \cdot g \cdot h
$$

$g$ is the gravitational constant.

\section{Pendulum Experiments}

A pendulum setup as described in detail in [20] was used to determine the maximal deflection velocity of the indenter $v_{A}$ and to estimate whether the LiSE indentation is in the quasistatic or high-speed range. The impact velocity is determined for the assembly with a cylindrical pressure cell and without a pressure cell. The shock wave is generated directly on the indenter with an indenter diameter of $D_{I}=3 \mathrm{~mm}$. The indenter is attached to a thread with a pendulum length of $l_{p}=450 \mathrm{~mm}$. The thread has a diameter of $d_{p}=0.09 \mathrm{~mm}$ and a tensile strength of $\sigma_{m}=7100 \mathrm{~N} / \mathrm{mm}^{2}$. Blind holes with a depth of $0.5 \mathrm{~mm}$ and a diameter of $0.2 \mathrm{~mm}$ are laser-drilled in the indenter with a nanosecond laser from IPG. The thread is glued inside the blind holes. The total mass $m_{G}$ is composed of the mass of the thread and the mass of the 
indenter. The kinetic energy of the laser-induced shock wave is transmitted on the indenter. The resulting deflection of the pendulum mass is recorded with the highspeed camera Phantom V5.1 from Vision Research. A frame rate of $1000 \mathrm{fps}$ with an exposure time of $990 \mu$ s is used. By calibrating the pixels via the diameter of the spherical indenter, a resolution of $0.077 \mathrm{~mm} / \mathrm{px}$ is determined. The kinetic energy $E_{k i n}$ is derived by the maximum deflection velocity of the indenter $v_{A}$. This is determined by extrapolating the measured deflection velocity of the indenter over time and calculating the $y$-axis intercept.

$$
E_{k i n}=\frac{1}{2} \cdot m_{G} \cdot v_{A}^{2}
$$

\section{Time-Resolved Force Measurement Setup}

\section{Measurement Setup}

Force measurements were performed with a piezoelectric polyvinylidene fluoride (PVDF) shock gauge sensor from Piezotech (piezo strain constant of $-22.0 \mathrm{pC} / \mathrm{N}$ ). The sensor is embedded in PTFE-foil and has an additional polyester protection on both sides (sensor model S_25CP). PVDF-sensors have a nanosecond resolution and are suitable for recording measurements with impact loading [21]. As shown in

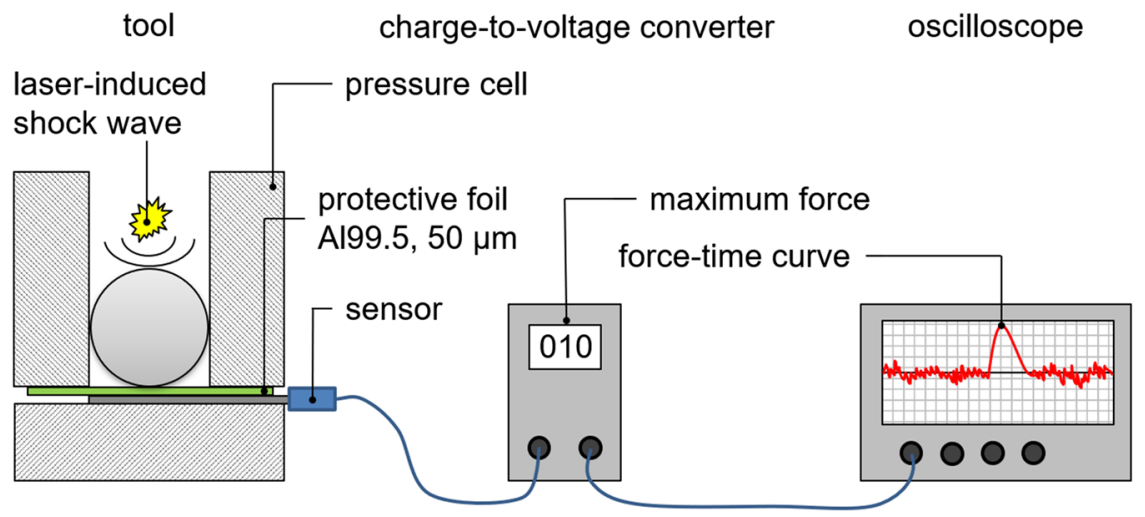

top view on shock gauge sensor

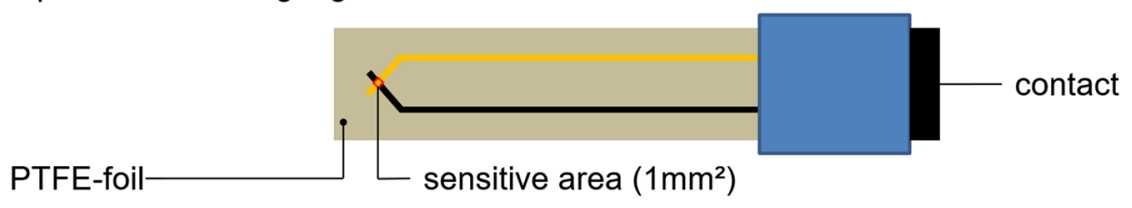

Valentino 2020

BIAS ID 201028

Fig. 4 Schematic setup for time-resolved force measurements for LiSE 
Fig. 4, the shock gauge sensor was placed below the indenter. The shock gauge sensor was fixated on a ceramic blank.

The sensor has a sensing area of $1 \mathrm{~mm}^{2}$, which was positioned coaxially to the laser beam and indenter. An A199.5 foil with a thickness of $50 \mu \mathrm{m}$ was placed between the indenter and PVDF shock gauge sensor as a protective foil to avoid damage of the sensor by the laser irradiation. The protective foil is also supposed to prevent excessive point loading and allow an accurate force measurement. To guarantee a contact between protective film and sensor with as little air gap as possible, the use of a hold-down device is necessary. The pressure cell functions as the hold-down device. The signal from the shock gauge was fed into the charge amplifier from Kistler (type 5015A). The charge amplifier possesses a sample rate of 1000000 data points per second with a bandwidth $(-3 \mathrm{~dB})$ of $200 \mathrm{kHz}$. To gain a time resolved force measurement, the charge amplifier was connected to a LeCroy waverunner LT374 oscilloscope. For every condition, 10 force measurements were conducted. The maximum force and transferred momentum were measured from each force-time profile. The transferred momentum is determined by the integration of the force-time curve between the first force increase and the maximum. Further force maxima are not considered within the integral because experiments revealed that the measured maximum force correlates with the indentation geometry [7]. The average of the determined values and the standard deviation were calculated.

\section{Theory}

Since there is a protective foil between the indenter and the sensor, it must be clarified whether the momentum and the maximum force transferred by the spherical indenter through the protective foil can be accurately measured and calculated based on the time-resolved force-measurement system. The validation of the time-resolved force-measurement system is performed in three steps:

- Firstly, it is tested whether the conditions of a quasistatic normal impact are fulfilled. In case of a quasistatic impact, the contact forces correspond to the static forces [22] and can be derived from the equation of motion. In case of a quasistatic normal impact, the measurements on the compression testing machine can be compared with those of LiSE.

- Secondly, the influence of the protective film is examined from a material engineering point of view by analyzing the effect of multiple indentations on the measured force-time curve and the transferred momentum at the same position. The measurement setup is not changed to ensure that the indenter always indents at the same position of the protective foil. It is suggested that the determined momentum must not change over the number of indentations. On the contrary, the maximum measured force must change after each indentation because the foil experiences deformation.

- Thirdly, it is presumed that the measured maximum force changes with an increasing number of indentations, up to the point where the foil can no longer be plastically deformed. At this point, the indenter presses into an elastic half space and the 
measured and derived (from the momentum) forces of the LiSE process are comparable with a conventional quasistatic indentation process. Thus, indentations are induced with the same time-resolved measurement setup with a compression testing machine and compared to LiSE. The impact of a rigid indenter into an elastic halfspace is comparable to the impact of two elastic indenters [22].

The impact velocity $v_{0}$ can be calculated from the transferred momentum $M$ and the mass of the indenter $m_{I}$.

$$
M=m_{I} \cdot v_{0}
$$

The impulse shape is implied to be ideal. Therefore, the following applies for the initial conditions

$$
t(\tau=0)=0, v(\tau=0)=v_{0},
$$

where $t$ is the indentation depth and $v$ the velocity. The maximum force $F_{\max }$ is derived by solving the equation of motion according to [22] with the initial conditions from Eq. (5).

$$
F_{\text {max }}=\left(\frac{125}{36} \cdot m_{I}^{3} \cdot v_{0}{ }^{6} \cdot R_{I} \cdot E^{* 2}\right)^{1 / 5}
$$

The effective Young's modulus $E^{*}$ is calculated using the material characteristics of the protective aluminum foil $\left(E_{A l}=70 \mathrm{GPa}, \nu_{A l}=0.34\right)[23]$.

$$
E^{*}=\frac{E}{1-v^{2}}
$$

To fulfill the condition of a quasistatic indentation, the impact velocity $v_{0}$ must be much smaller than the characteristic propagation velocity of elastic waves $v_{e l}$ in the elastic half space [22]. According to Hunter, it is sufficient that the following relationship is fulfilled [24]:

$$
v^{\prime}=\left(\frac{v_{0}}{v_{e l}}\right)^{3 / 5} \ll 1
$$

The characteristic propagation velocity of elastic waves $v_{e l}$ correlates with the Young's modulus and the density of the material. For aluminum, the density is $\rho_{A l}=2.7 \mathrm{~g} / \mathrm{cm}^{3}$.

$$
v_{e l}=\sqrt{\frac{E}{\rho}}
$$

The impact velocity is derived from pendulum experiments. The impact velocity $v_{0}$ corresponds to the maximum deflection velocity of the pendulum $v_{A}$. 


$$
E_{K i n}=\frac{1}{2} \cdot m_{I} \cdot v_{0}^{2}=\frac{(M)^{2}}{2 \cdot m_{I}}
$$

\section{Results}

\section{Validation of Time-Resolved Force Measurement Method}

Figure 5 shows the time-resolved force measurement below the indenter for the cylindrical pressure cell in comparison with the reference setup (without pressure cell). The profile of the reference setup (without pressure cell) reveals a double-peak profile. In contrast, the force-time curve of the cylindrical pressure cell displays one strong maximum and then decreases down to zero after $20 \mu$ s. For each measured force-time curve, the signal-to-noise ratio of the charge-voltage amplifier results in a standard deviation of $U=0.3 \mathrm{~N}$.

The determined maximum deflection velocity is shown in Fig. 6 for the assembly with a cylindrical pressure cell and without a pressure cell. The maximum deflection speed is observed with the cylindrical cell.

Figure 7 shows the development of measured maximum force and the determined momentum as well as the maximum force calculated from Eq. (6) in dependence of the number of indentations at the same position of the protective foil. The measured maximum force increases with increasing number of indentations. This behavior is explained by the deformation of the protective foil with each indentation. With increasing number of indentations, the deformed indentation volume by the indenter decreases, which also decreases forming energy and accordingly, increases the measured maximum force. After 100 indentations, the measured maximum force approaches a value of $F_{\text {max }, m e}=27.7 \mathrm{~N}$. A standard deviation of $\Delta F_{\max }=0.5 \mathrm{~N}$ is measured in this range. The measurement of the momentum shows no significant change in dependence of indentations. On average, a momentum of $M=97.7 \mu$ Ns is

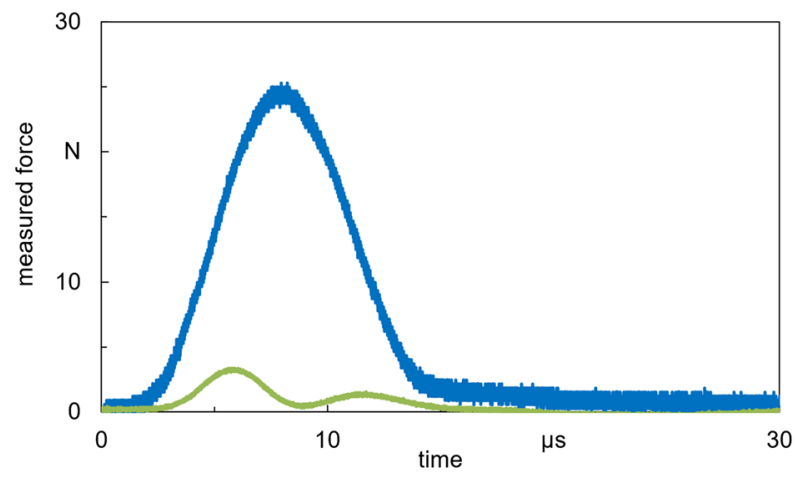

Valentino 2019

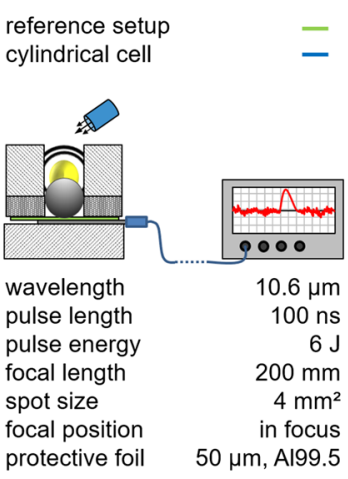

BIAS ID 191201

Fig. 5 Measured force-time curves of tested pressure cells 
Fig. 6 Determination of the pendulum speed by means of the pendulum test with and without pressure cell

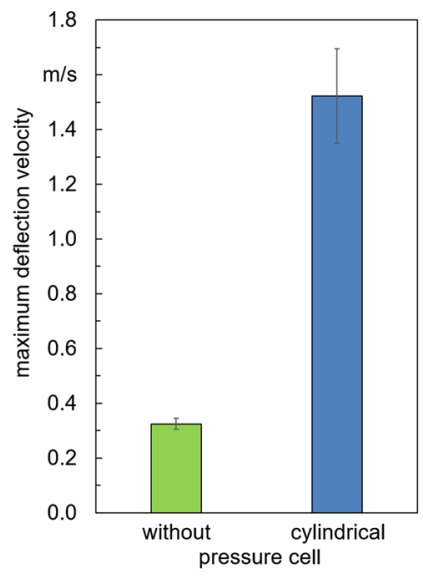

pulse energy

focal lengh

focal position

intensity

indenter diameter

indenter material
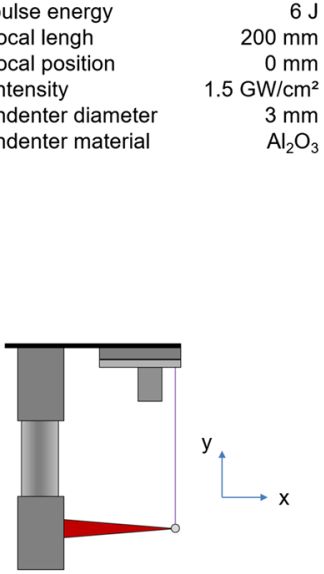

BIAS ID 201029
$/ \mathrm{cm}^{2}$

$\mathrm{Al}_{2} \mathrm{O}_{3}$

calculated, with a standard deviation of $\Delta M=5.4 \mu N s$. From the momentum a maximum force of $F_{\text {max }, c a l}=40.9 \mathrm{~N} \pm 2.7 \mathrm{~N}$ is calculated. The deviation of the measured maximum force $F_{\text {max }, \text { me }}$ from the calculated maximum force $F_{\text {max } \text {, cal }}$ is $30 \%$.

Moreover, the change in indentation diameter and indentation depth is plotted against the number of indentations. Figure 8 shows that the change of the indentation depth and the indentation diameter decreases with increasing number of indentations. After 90 indentations, no significant change of the indentation diameter and the indentation depth is observed.

For indentations by means of a compression testing machine, the same indenter is attached to a die and pressed into the piezoelectric sensor with protective foil. A defined force of $F_{\text {max }}=30 \mathrm{~N}$ is applied with the tensile-compression testing machine

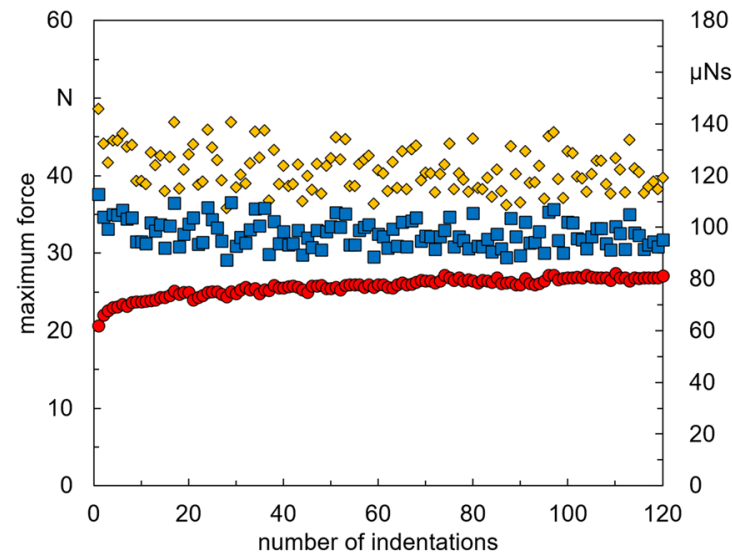

measured maximum force calculated maximum force momentum

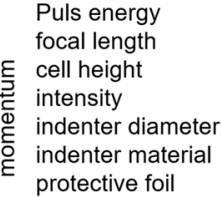

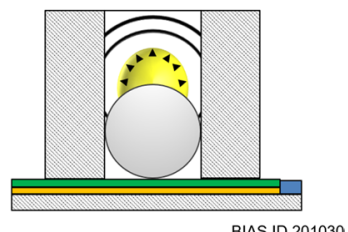

Valentino 2020

Fig. 7 Development of the measured maximum force, the transferred momentum, and the calculated maximum force over the number of indentations 


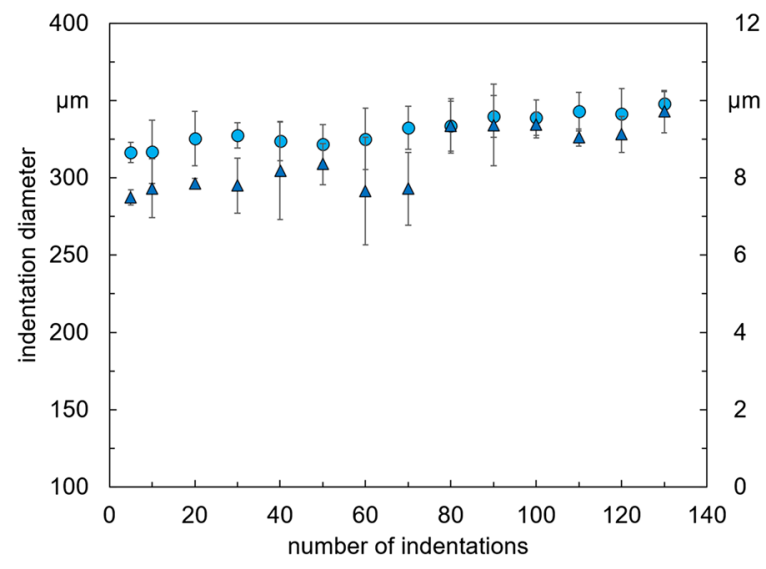

indentation diameter indentation depth

$8 \neq$ cell height

Valentino 2020

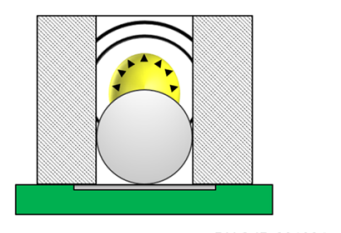

Fig. 8 Development of the induced indentation depth in the protective foil over the number of indentations at the same position

Z250. In Fig. 9 the first measured value is minimally lower than the maximum force set at the compression testing machine. With increasing number of indentations, the measured maximum force is reduced until a plateau is reached after 70 indentations. The relative deviation between set maximum force and measured maximum force is $30 \%$ in this range.

Additionally, the maximum force $F_{I}$ is varied at the compression testing machine and compared to the measured maximum force $F_{G}$ at the pressure sensor (see Fig. 10). The indenter is pushed in same position as before. Three measurements are performed at each set maximum force. Only a small scattering is detected for each measured maximum force. Between the set maximum
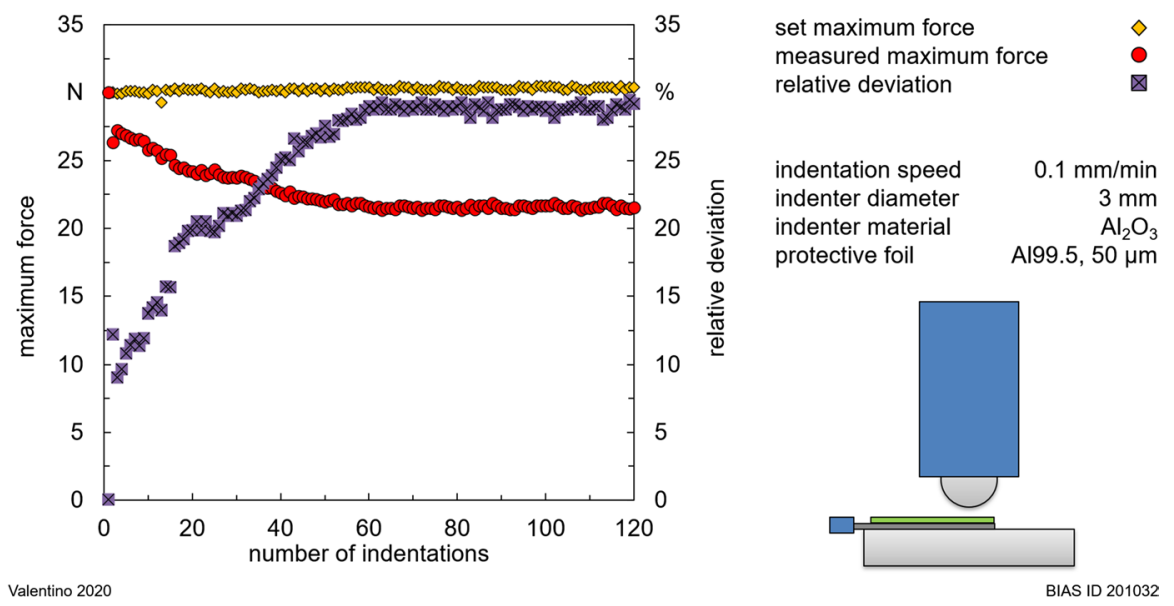

Valentino 2020

Fig. 9 Development of the maximum force $F_{G}$ measured with piezo sensor in comparison to the set maximum force $F_{I}$ with conventional testing machine 


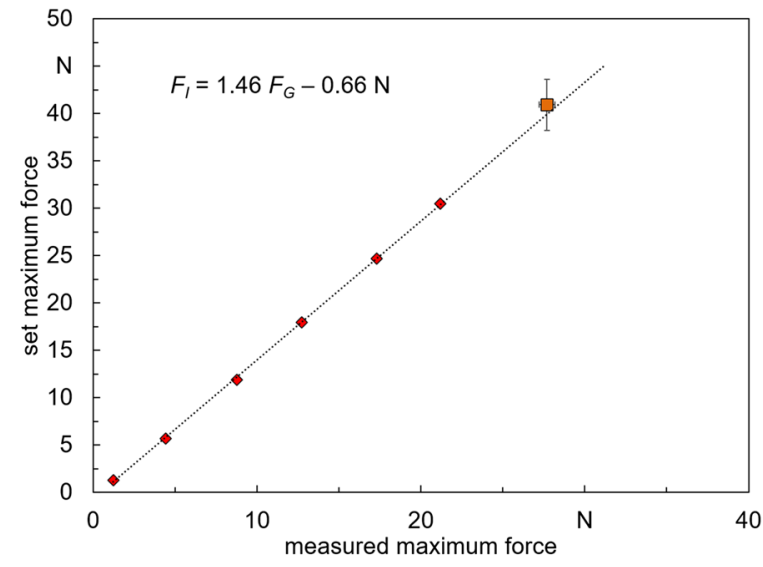

Z250 testing machine

LiSE force measurements trend line

$\mathbf{Z 2 5 0}$

indentation speed

$0.1 \mathrm{~mm} / \mathrm{min}$

LiSE

pulse energy

focal length

cell height

intensity

$5.5 \mathrm{~J}$

$200 \mathrm{~mm}$

$10 \mathrm{~mm}$

indenter diameter $\quad 3 \mathrm{~mm}$

indenter material protective foil
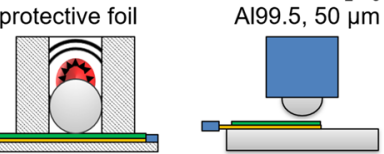

Valentino 2020

BIAS ID 201033

Fig. 10 Difference between the measured maximum force $F_{G}$ with piezo sensor and the set maximum force $F_{I}$ with conventional testing machine and LiSE

force and the measured force, a linear trend is obtained, from which a calibration curve $F_{I}\left(F_{G}\right)$ is derived. When this linear trend line is continued, the calculated maximum force in dependence of the measured maximum force of LiSE lies on the trend line.

Moreover, indentations were induced in A199.5 and the steel S235 and compared with the drop test with the LiSE. Figure 11 compares the measurement results of the drop test with those of LiSE. The drop height was varied, and a trend was determined. For LiSE a pulse energy of $E_{P}=6 \mathrm{~J}$ and a focal position of $f_{p}=+10 \mathrm{~mm}$ were used. For both methods, no significant differences can be obtained between the indentation volume induced with LiSE and the indentation
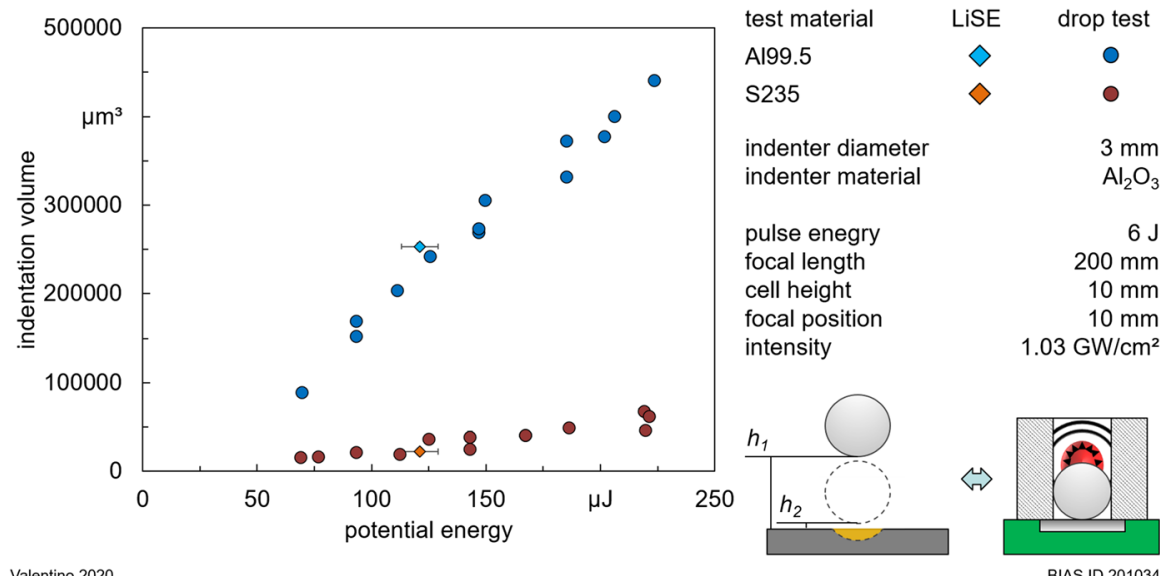

Valentino 2020

Fig. 11 Comparison between drop tests and LiSE of the induced indentation volume 
volume induced with drop tests at similar potential energy. This is behavior also observed for both investigated materials.

\section{Determination of Position Tolerances}

\section{Force and Momentum Measurements}

The distribution of maximum force does not show significant changes between the shift in $\mathrm{x}$ - and $\mathrm{y}$-direction. Thus, the mean value was taken from the two respective values in $\mathrm{x}$ - and $\mathrm{y}$-direction and referred to as lateral shift, as shown in Fig. 12. By positioning the laser beam away from the center of the indenter, the maximum transmitted force and momentum on the indenter is decreased. Moreover, the standard deviation increases with increasing lateral shift. A significant decrease in maximum force and momentum is observed at the lateral shift of $\Delta x= \pm 0.5 \mathrm{~mm}$.

For the cylindrical pressure cell, the shift of focal position $f_{p}$ increases the acting maximum force until $f_{p}=-4 \mathrm{~mm}$ and $f_{p}=12 \mathrm{~mm}$ (see Fig. 13). Further shifting the focus away from the surface leads to decrease in transmitted force and momentum. By shifting the focus inside the indenter $\left(f_{p}<0\right)$, the increase in force and momentum is not as dominant compared to the shift in focal position above the indenter.

\section{Indentations}

Figure 14 shows indentations measured at $20 \times$ magnification with the microscope VK9710 from Keyence. The reference position in focus and center is compared to different focal positions and lateral shifts.

In Fig. 15, the influence of lateral shift is shown. The shift leads to a decrease in induced indentation diameter and indentation depth (see Fig. 15). A significant decrease in indentation diameter is observed at a lateral shift of $\Delta x=0.5 \mathrm{~mm}$. Moreover, the lateral shift leads to an increase in standard deviation.

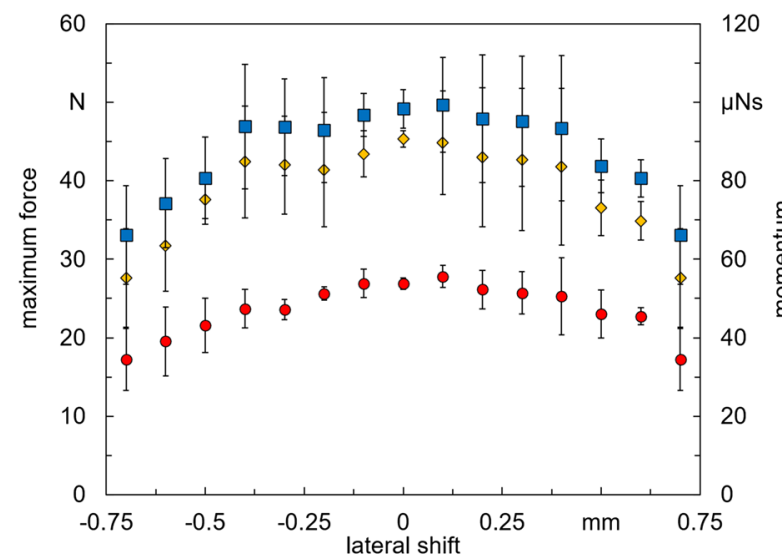

Valentino 2019 measured maximum force calculated maximum force momentum

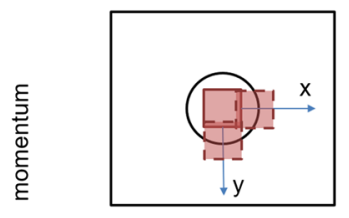

wavelength pulse length pulse energy focal length spot size focal position protective foil
$10.6 \mu \mathrm{m}$ $100 \mathrm{~ns}$

$6 \mathrm{~J}$

$200 \mathrm{~mm}$

$4 \mathrm{~mm}^{2}$

in focus

$50 \mu \mathrm{m}$, Al99.5

Fig. 12 Influence of lateral shift on maximum transmitted force and momentum on the indenter 


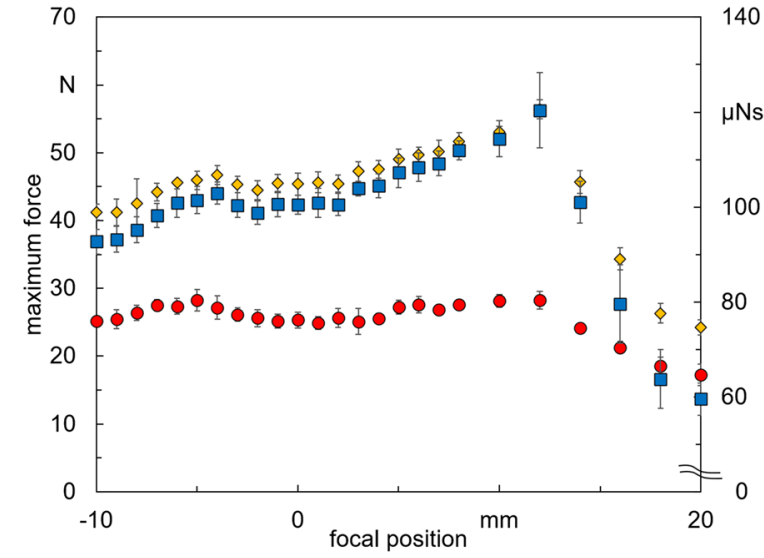

Valentino 2019 measured maximum force calculated maximum force momentum

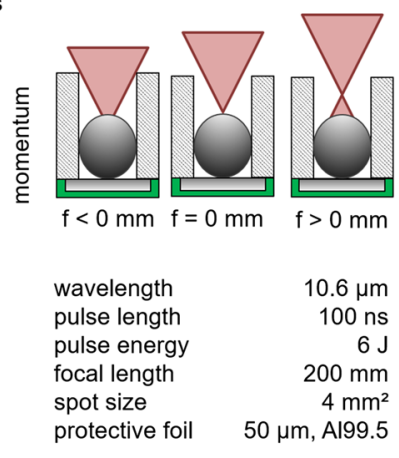

Fig. 13 Influence of focal position on transmitted force and momentum on indenter

Figure 16 shows the influence of shift in focal position on indentation geometry for the cylindrical pressure cell. The measured indentation diameter increases when the focus is shifted above or inside the indenter surface. A significant change is observed at the focal positions $f_{p} \geq-3 \mathrm{~mm}$ and $f_{p} \leq 2 \mathrm{~mm}$ in reference to the position in focus $\left(f_{p}=0\right)$.

\section{Discussion}

The derived maximum deflection velocities in Fig. 5 reveal that the conditions of a quasistatic normal impact are fulfilled according to the findings of Hunter [24]. Since the highest deflection velocities are observed with the cylindrical pressure cell, it

Fig. 14 Exemplary indentations demonstrating the influence on indentation geometry for different focal positions and lateral shifts
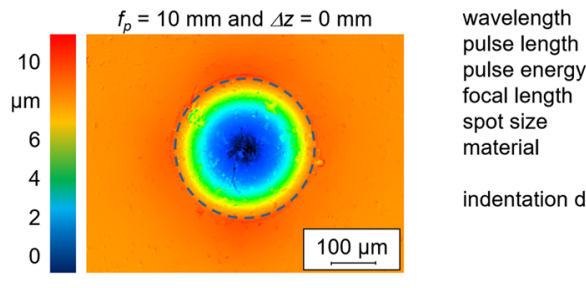

$10.6 \mu \mathrm{m}$ $100 \mathrm{~ns}$

$6 \mathrm{~J}$

$200 \mathrm{~mm}$

$4 \mathrm{~mm}^{2}$ Al99.5

indentation diameter
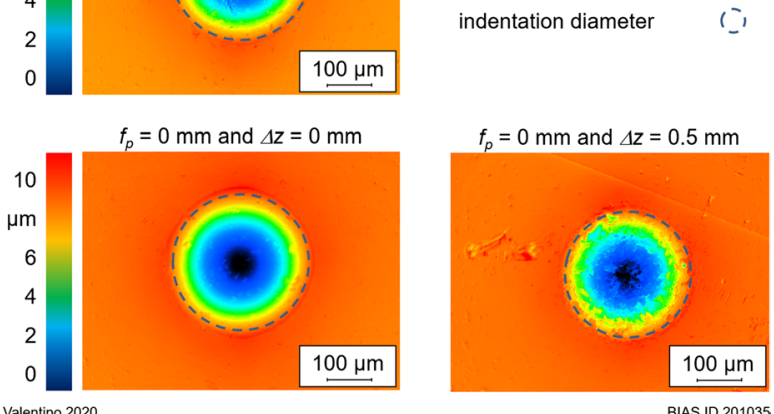


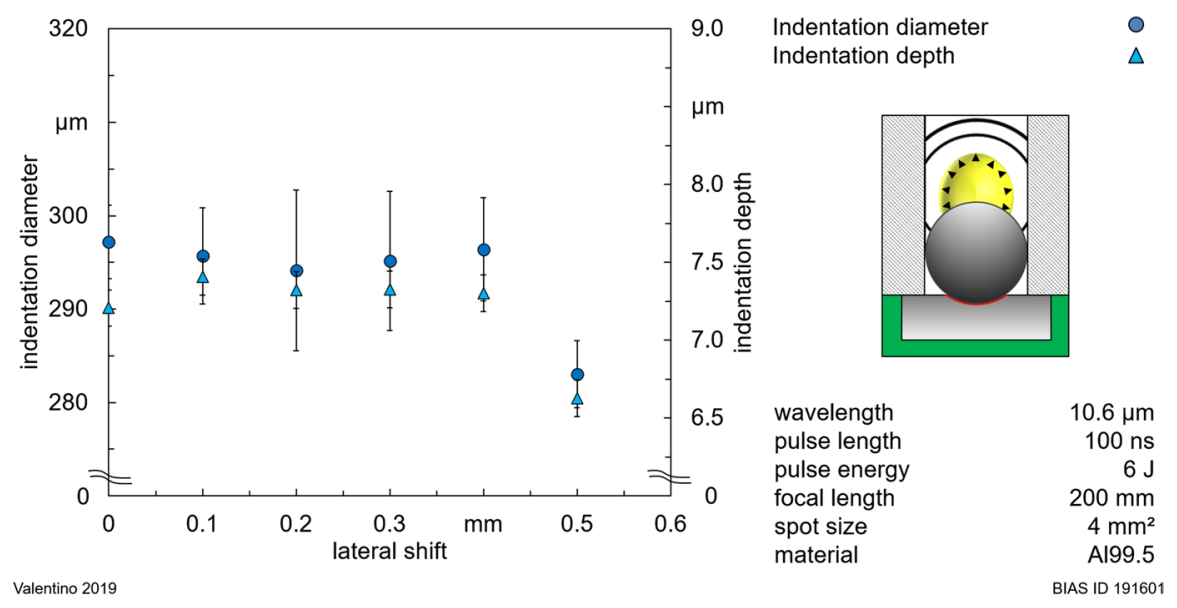

Fig. 15 Influence of lateral shift on indentation diameter and indentation depth

can be implied that this cell also represents a critical velocity for the validity of the quasistatic indentation. With Eq. (8), the velocity ratio $v$ ' of the pendulum and the characteristic velocity of elastic waves in aluminum is calculated. The ratio without a pressure cell is $v_{o}^{\prime}=0.003$ and the ratio with a cylindrical pressure cell $v_{D R Z}^{\prime}=0.008$. Both ratios are clearly below $v^{\prime}{ }_{\max }<<1$, which indicates that the condition of a quasistatic indentation is fulfilled. Thus, the LiSE indentation process is quasistatic, wherefore the present contact forces correspond with the static ones. When the linear trend line is continued in Fig. 9, the calculated maximum force in dependence of the measured maximum force of LiSE lies on the trend line, which validates the derived maximum force. Still, it must be considered that the indentation behavior might differ

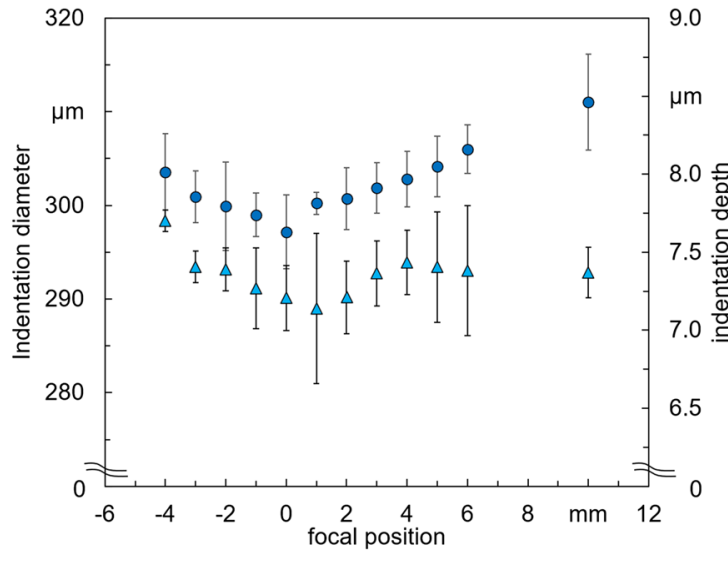

Valentino 2019
Indentation diameter

Indentation depth

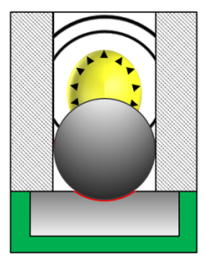

wavelength pulse length pulse energy

focal length spot size material
$10.6 \mu \mathrm{m}$ $100 \mathrm{~ns}$

$6 \mathrm{~J}$

$200 \mathrm{~mm}$ $4 \mathrm{~mm}^{2}$ Al99.5

BIAS ID 191602

Fig. 16 Influence of focal position on indentation diameter and indentation depth 
for impulse-based indentation processes in dependence of the material properties such as young's modulus and strain hardening coefficient. Hence, it is important that the rebound speed must be additionally measured to derive the acting forces, which are relevant for plastic deformation. Consequently, the determined maximum forces from the measurement method cannot be compared with the Brinell hardness from Eq. (1) without additional data about the rebound behavior.

No significant differences can be obtained in Fig. 11 between the indentation volume induced with LiSE and the trend of the indentation volume induced with drop tests, which additionally validates the determined momentum with the time-resolved measurement setup. It is demonstrated that the measured maximum force is influenced in dependence of the number of indentations (see Fig. 8 and Fig. 9). However, the transmitted momentum and the derived maximum force from Eq. (6) remain constant. Thus, the introduced time-resolved measurement system is valid to obtain the momentum of the indenter and maximum transmitted maximum force of the indenter on the workpiece. The validation of the measurement setup allows to use the maximum force or momentum as a reference value and to derive position tolerances.

A shift of the focal position away from the focus (reference position) leads to an increase in maximum transmitted force in both directions (see Fig. 13). The indentation experiments in Fig. 16 underline these findings. When shifting the focal position, the increase in maximum force can be explained by plasma plume investigations shown in [25]: The origin of the shock wave is shifted closer to the indenter surface. Additionally, the pulse energy predominantly governs the shock wave propagation velocity [26]. Both mechanisms increase the transmitted force on the indenter. By shifting the focus inside the indenter $\left(f_{p}<0\right)$, the increase in maximum force and momentum is not as dominant compared to the shift in focal position above the indenter. When the focus is shifted inside the indenter $\left(f_{p}<0\right)$, the laser beam partially irradiates on the upside of the pressure cell (height $10 \mathrm{~mm}$ ). This causes the formation of a plasma on top of the pressure cell, which absorbs the laser irradiation. However, this effect cannot be accounted for the decrease in maximum force when the focus is shifted above the indenter $\left(f_{p}>12 \mathrm{~mm}\right)$. The beam diagonal is not large enough to interact with the upside of the pressure cell. The decrease in maximum force can be explained by the Brewster angle $\theta_{B}$ of the pressure cell made of brass in dependence of the laser wavelength. The Brewster angle is $\theta_{B}=89^{\circ}$ [27] for the wavelength of the laser and brass. This Brewster angle is in the range of the angle of incidence of the laser beam on the inside wall of the pressure cell when the focal position is shifted above the indenter. Thus, the shock wave is created further away from the indenter surface, which decreases the transmitted force on the indenter.

Regarding the positioning tolerances of the vertical displacement (shift in focal position), we obtain acceptable positioning tolerances between $f_{p} \geq-3.0 \mathrm{~mm}$ and $f_{p} \leq+2.0 \mathrm{~mm}$ for the calculated maximum forces in Fig. 13 and the measured indentation diameter in Fig. 16. The relatively large vertical positioning tolerances are attributed to the Rayleigh length of the laser beam $\left(z_{R}=10.8 \mathrm{~mm}\right)$. Moreover, no differences are obtained for positioning tolerances of the lateral shift between the calculated maximum forces in Fig. 12 and the measured indentation diameter in Fig. 15. A lateral shift of the laser beam leads to a changed beam profile on the surface. The resulting maximum force as well as indentation diameter do not seem to 
change significantly up to a lateral shift of $\Delta x= \pm 0.4 \mathrm{~mm}$ (see Fig. 12 and Fig. 15). The cylindrical pressure cell seems to compress the plasma formation to a small location above the indenter. Only when the plasma formation absorbs a large amount of the laser irradiation (like the shift in focus inside the indenter), the maximum force transmitted on the indenter is reduced.

\section{Conclusions}

A measurement method was introduced to determine the momentum and maximum forces transmitted on spherical indenters by laser-induced shock waves. The influence of eccentric irradiation and shift in focal position were investigated with this method to determine the required positioning tolerances. The conclusions are summarized as follows:

- The introduced time-resolved measurement system is valid to obtain the momentum of the indenter and maximum transmitted force of the indenter.

- From the validated time-resolved force measurement method and indentation experiments we derive that deviations from the maximum forces are still acceptable, when the lateral deviation of the beam center, which depends only on the alignment of the setup, does not exceed $\pm 0.4 \mathrm{~mm}$. The rather small lateral positioning tolerances are caused by plasma shielding above the cylindrical pressure cell. A vertical displacement from the focus position between $f_{p} \geq-3.0 \mathrm{~mm}$ and $f_{p} \leq+2.0 \mathrm{~mm}$ still leads to acceptable deviations from the indentation force. The larger vertical positioning tolerances are attributed to the large Rayleigh length of the laser beam.

- The LiSE indentation process is quasistatic, wherefore the present contact forces correspond with the static ones. However, the indentation behavior differs for impulse-based indentation processes in dependence of the material properties such as young's modulus and strain hardening coefficient. Thus, the rebound speed must be additionally measured to derive the acting forces during plastic deformation.

Acknowledgement Financial support of the subproject D02 "Laser induced hardness measurements" funded by the Deutsche Forschungsgemeinschaft (DFG, German Research Foundation) - Project number 276397488 - SFB 1232 is gratefully acknowledged.

Funding Open Access funding enabled and organized by Projekt DEAL.

Open Access This article is licensed under a Creative Commons Attribution 4.0 International License, which permits use, sharing, adaptation, distribution and reproduction in any medium or format, as long as you give appropriate credit to the original author(s) and the source, provide a link to the Creative Commons licence, and indicate if changes were made. The images or other third party material in this article are included in the article's Creative Commons licence, unless indicated otherwise in a credit line to the material. If material is not included in the article's Creative Commons licence and your intended use is not permitted by statutory regulation or exceeds the permitted use, you will need to obtain permission directly from the copyright holder. To view a copy of this licence, visit http://creativecommons.org/licen ses/by/4.0/. 


\section{References}

1. Kaufman, L., Bernstein, H.: Computer Calculation of Phase Diagrams with Special Reference to Refractory Metals. University of Michigan; Academic Press (1970)

2. Bensch, M., Schulze Wierling, P., von Lieres, E., Hubbuch, J.: High throughput screening of chromatographic phases for rapid process development. Chem. Eng. Technol. 28, 1274-1284 (2005)

3. Mädler, L.: Is High-throughput screening for structural materials / metals possible? In Fang, F., Brinksmeier, E., Riemer, O. (eds.) Proceedings of the 4th International Conference on Nanomanufacturing, Bremen. 1-3 (2014)

4. Potyrailo, R., Rajan, K., Stoewe, K., Takeuchi, I., Chisholm, B., Lam, H.: Combinatorial and high-throughput screening of materials libraries: review of state of the art. ACS Comb. Sci. 13, 579-633 (2011)

5. Ellendt, N., Mädler, L.: Hochdurchsatzexploration evolutionärer Konstruktionswerkstoffe. HTM J. Heat Treatm. Mat. 73, 3-12 (2018)

6. Steinbacher, M., Alexe, G., Baune, M., Bobrov, I., Bösing, I., Clausen, B., Czotscher, T., Riemer, O., Sonnenberg, H., Thomann, A., Toenjes, A., Vollertsen, F., Wielki, N., Ellendt, N.: Descriptors for high throughput in structural materials development. High-throughput 8, 2-27 (2019)

7. Fenske, H., Czotscher, T.: Tailoring the pressure profile of TEA-CO2 laser-induced shock waves for mechanical forming and separation processes. Lasers Manuf. Mater. Process. 7, 1-14 (2019). https:// doi.org/10.1007/s40516-019-00105-y

8. Czotscher, T., Baguer, D.O., Vollertsen, F., Piotrowska-Kurczewski, I., Maaß, P.: Connection between shock wave induced indentations and hardness by means of neural networks. AIP Conf. Proc. 2113, 1-6 (2019)

9. Czotscher, T., Von Hehl, A., Radel, T., Toenjes, A.: Correlation between Shock Wave-induced Indentations and Tensile Strength. Procedia Manuf. 47, 756-760 (2020)

10. Bergmann, H.W.: Hügel H (1998) Modellierung und Diagnostik des Abtragsprozesses. Strahltechnik 6, 35-44 (1998)

11. Radziemski, L.J., Cremers, D.A., Niemczyk, T.M.: Measurement of the properties of a $\mathrm{CO}_{2}$ laser induced air-plasma by double floating probe and spectroscopic techniques. Spectrochim. Acta 40B, 517-525 (1985). https://doi.org/10.1016/0584-8547(85)80089-6

12. Czotscher, T., Vollertsen, F.: Proceedings of the 8th International Conference on High Speed Forming, Columbus Ohio, 14-16 May 2018. (2018). https://doi.org/10.17877/DE290R-18958

13. Nielsen, P.E.: Hydrodynamic calculations of surface response in the presence of laser-supported detonation waves. J. Appl. Phys. 46, 4501-4505 (1975)

14. Barchukov, A.I., Bunkin, F.V., Konov, V.I., Lyubin, A.A.: Investigation of Low-Threshold Gas Breakdown Near Solid Targets by $\mathrm{CO}_{2}$ Laser Radiation. Sov. Phys. 39, 42-45 (1974)

15. Sai Shiva, S., Leela, C., Chaturvedi, S., Sijoy, C.D., Prem Kiran, P.: Development of numerical model to investigate the laser driven shock waves from aluminum target into ambient air at atmospheric pressure and its comparison with experiment. AIP Conf. Proc. 1793, 1-5 (2017). https://doi.org/10.1063/1. 4971619

16. Corsi, M., Cristoforetti, G., Hidalgo, M., Iriarte, D., Legnaioli, S., Palleschi, V., Salvetti, A., Tognoni, E.: Effect of laser-induced crater depth in laser-induced breakdown spectroscopy emission features. Appl. Spectrosc. 59, 853-860 (2005)

17. Czotscher, T., Veenaas, S., Vollertsen, F.: Possibilities to characterise laser induced shockwaves. J. Technol. Plast. 42, 1-7 (2017). https://doi.org/10.24867/jtp.2017.42-1.1-7

18. DIN 51200:2016-08: Werkstoffprüfmaschinen - Gestalten und Anwenden von Aufnahmevorrichtungen für Proben in Härteprüfgeräten. (2016)

19. DIN EN ISO 6506-1:2015-02: Metallische Werkstoffe - Härteprüfung nach Brinell - Teil 1: Prüfverfahren. (2015)

20. Czotscher, T.: Material Characterisation with New Indentation Technique Based on Laser-Induced Shockwaves. Lasers Manuf. Mater. Process. 5, 439-457 (2018)

21. Bauer, F.: PVDF shock compression sensors in shock wave physics. AIP Conf. Proc. 706, 1121-1124 (2004). https://doi.org/10.1063/1.1780435

22. Willert, E.: Stoßprobleme in Physik, Technik und Medizin. Springer Vieweg (2020)

23. Popov, V.L.: Kontaktmechanik und Reibung. Springer-Verlag (2009)

24. Hunter, S.C.: Energy Absorbed by Elastic Waves During Impact. J. Mech. Phys. Solids 5, 162-171 (1957)

25. Czotscher, T.: Analysis of TEA-CO $\mathrm{CO}_{2}$-laser-induced plasma to establish a new measurement technique. J. Laser Appl. 30, (2018). https://doi.org/10.2351/1.5040600 
26. Zel'dovich, Y.B., Raizer, Y.P., Hayes, W.D., Probstein, R.F., Landshoff, R.: Physics of Shock Waves and High-Temperature Hydrodynamic Phenomena, vol. 2. Academic Press, New York (1966)

27. Querry, M.R.: Optical constants, Contractor Report CRDC-CR-85034 (1985)

Publisher's Note Springer Nature remains neutral with regard to jurisdictional claims in published maps and institutional affiliations. 